\title{
Analysis and functional annotation of expressed sequence tags from the fall armyworm Spodoptera frugiperda Youping Deng1,2, Yinghua Dong ${ }^{1}$, Venkata Thodima ${ }^{2}$, Rollie J Clem ${ }^{1}$ and A Lorena Passarelli*1
}

\author{
Address: ${ }^{1}$ Molecular, Cellular, and Developmental Biology Program, Division of Biology, Kansas State University, Manhattan, Kansas 66506, USA \\ and ${ }^{2}$ Department of Biological Sciences, The University of Southern Mississippi, Hattiesburg, MS 39402, USA \\ Email: Youping Deng - youping.deng@usm.edu; Yinghua Dong - yinghua@ksu.edu; Venkata Thodima - venkata.thodima@usm.edu; \\ Rollie J Clem - rclem@ksu.edu; A Lorena Passarelli* - lpassar@ksu.edu \\ * Corresponding author
}

Published: 19 October 2006

BMC Genomics 2006, 7:264 doi:10.1 |86/|47|-2|64-7-264
Received: 28 March 2006

Accepted: 19 October 2006

This article is available from: http://www.biomedcentral.com//47/-2/64/7/264

(c) 2006 Deng et al; licensee BioMed Central Ltd.

This is an Open Access article distributed under the terms of the Creative Commons Attribution License (http://creativecommons.org/licenses/by/2.0), which permits unrestricted use, distribution, and reproduction in any medium, provided the original work is properly cited.

\begin{abstract}
Background: Little is known about the genome sequences of lepidopteran insects, although this group of insects has been studied extensively in the fields of endocrinology, development, immunity, and pathogen-host interactions. In addition, cell lines derived from Spodoptera frugiperda and other lepidopteran insects are routinely used for baculovirus foreign gene expression. This study reports the results of an expressed sequence tag (EST) sequencing project in cells from the lepidopteran insect $S$. frugiperda, the fall armyworm.
\end{abstract}

Results: We have constructed an EST database using two cDNA libraries from the $S$. frugiperdaderived cell line, SF-2I. The database consists of 2,367 ESTs which were assembled into 244 contigs and $95 \mathrm{I}$ singlets for a total of $\mathrm{I}, 195$ unique sequences.

Conclusion: S. frugiperda is an agriculturally important pest insect and genomic information will be instrumental for establishing initial transcriptional profiling and gene function studies, and for obtaining information about genes manipulated during infections by insect pathogens such as baculoviruses.

\section{Background}

The nucleotide sequences from numerous animal genomes have been derived and include examples from both vertebrate and invertebrate organisms. In addition, determination of the genomic sequences of many more organisms are in progress, yielding a broad picture of the diversity and common pathways among differing organisms. Genome sequences for the insects Apis mellifera (honeybee), Anopheles gambiae (mosquito), Drosophila melanogaster (fruit fly), and Bombyx mori (silkworm) have been reported [1-5], and additional insect genome sequences including Acyrthosiphon pisum (pea aphid), Aedes aegypti and Culex pipiens (mosquitoes), several Drosophila species, Nasonia vitripennis (parasitoid wasp), Rhodnius prolixus (insect vector for Trypanosoma cruzi), and Tribolium castaneum (red flour beetle) are anticipated. In addition, a number of EST databases derived from Lepidoptera are available (NCBI dbEST). The Lepidoptera (moths and butterflies) are the second largest order of the class Insecta. As such, they are a diverse group of insects distributed worldwide and throughout different climates ranging from that in Siberia to the tropics. Thus, it is 
important to compare the genomes of a number of species within the order and to that of other insects.

Lepidoptera are viewed as being among the most beautiful insects, yet their larvae are major pests to economically important crops and forests. Among the Lepidoptera, the silkworm, B. mori, has been studied intensively, since it is a commercially important insect. In addition, the Lepidoptera are valued as models to examine insect-plant and insect-pathogen interactions. Their study and comparative genomic analyses will render valuable tools for insect pest management and the improvement of widely used lepidopteran pathogens, the baculoviruses, as foreign gene expression vectors.

In this study, we report the establishment of an expressed sequence tag (EST) database of 1,195 unique sequences from the cell line IPLB-SF-21 (SF-21) [6], a cell line derived from immature ovaries of pupae of the fall armyworm, Spodoptera frugiperda. This and other EST databases can serve as the starting point from which other $S$. frugiperda genome clones can be surveyed or to explore gene expression profiles using microarray assays. More importantly, these and additional ESTs can be the basis for comparative genomic analyses among Lepidoptera or other genomes within the Insecta.

\section{Results}

\section{General sequence survey}

To start characterizing SF-21 sequences, two independent directionally cloned cDNA libraries that had been previously constructed for use in yeast two-hybrid screens using the plasmid vectors $\mathrm{pB} 42 \mathrm{AD}$ and pYES2 (Invitrogen) were utilized. Initially, about 200 clones were partially sequenced from each library to assess library quality. Both libraries yielded acceptable results. The library cloned in pYES2 was selected for further sequencing. In all, a total of 3,365 cloned inserts were subjected to single-pass sequencing from their 5 ' ends, 192 clones in the vector pB42AD and 3,173 clones in the vector pYES2. The 3,365 sequences were trimmed of vector sequences, poly $\mathrm{A} / \mathrm{T}$ tails, low quality, adaptor, and contaminating bacterial sequences, and screened for a minimum length of $200 \mathrm{bp}$. This resulted in a total of 2,367 high quality ESTs with an average length of 610 bases ( 156 sequences from pB42AD and 2,211 sequences from pYES2). No attempt was made to carry out sequencing to saturation. These ESTs were assembled using the CAP3 [7] program and verified using the Phrap [8] program. Both programs assemble overlapping ESTs to commence forming contigs. A total of 1,417 ESTs were assembled into 244 contigs, leaving 951 sequences as singlets. Contigs and singlets together resulted in 1,195 unique sequences that putatively represent different transcripts. The number of ESTs in the 244 contigs varied from 2 to $63 ; 56 \%$ of contig sequences had two ESTs, $10 \%$ had three ESTs, and $10 \%$ had greater than 10 ESTs (Fig. 1). The average length of the assembled contigs was 854 bases; longer than the average length of singlets ( 617 bases). The longest contig sequence, contig 138, was 2,361 bases.

\section{Highly redundant genes}

A total of 14 contigs containing 307 ESTs were highly redundant. This accounted for more than $13 \%$ of the total high quality ESTs. The minimum number of ESTs that made up these most highly redundant contigs was 13 (Table 1). Distribution of ESTs in each contig can be accessed via the ESTMD database [9] using the contig viewer search function. The best matched genes of 6 contigs, totaling 138 ESTs, are from $S$. frugiperda, verifying the source of the cDNAs. Nearly half (6) of the highly redundant contigs, totaling 90 ESTs, had significant homology to various ribosomal proteins, indicating high transcript abundance of ribosomal protein genes, as expected. Four contigs totaling 89 ESTs had matches similar to sequences derived from mitochondrial cytochrome b or cytochrome oxidase subunits (Table 1 ). The most redundant contig was composed of 63 ESTs and had significant homology to NADH dehydrogenase subunit -1 (ND-1) from S. frugiperda [10].

Percentage of contigs with number of ESTs

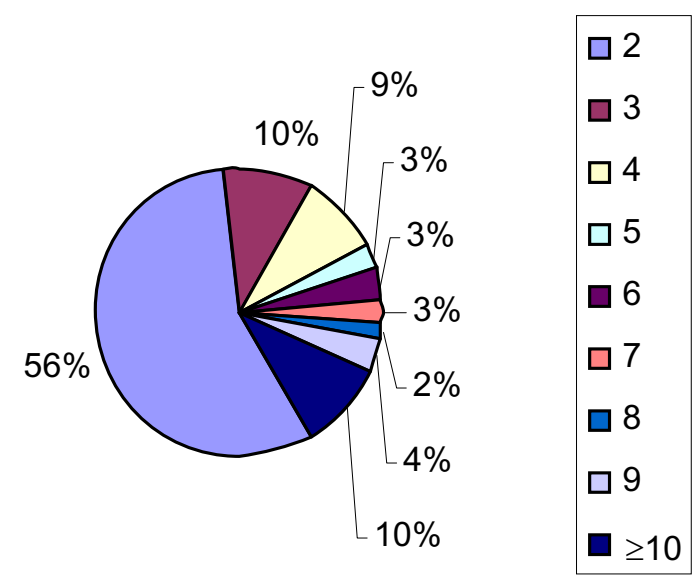

\section{Figure I}

Distribution of S. frugiperda ESTs. Percentage distribution of contig sequences with number of ESTs. The colorcoded legend indicates the number of ESTs in the contig sequences. 


\section{Comparative sequence analysis of S. frugiperda cDNA data}

We used the 1,195 unique ESTs to search non-redundant protein databases using BLASTX (Table 2). A total of 724 sequences $(60.6 \%)$ matched known proteins at a cut-off expectation (E)-value of $10^{-5}$ or below. Eleven sequences $(0.9 \%)$ had hits with E-values at $\mathrm{E}<10^{-150}, 53$ sequences (4.4\%) had hits with E-values between $10^{-150}$ and $10^{-100,}$ 283 sequences $(23.6 \%)$ had hits with E-values between $10^{-100}$ and $10^{-50}, 237$ sequences (19.8\%) had hits with Evalues between $10^{-50}$ and $10^{-20}$, and 140 sequences $(12 \%)$ had hits with E-values between $10^{-20}$ and $10^{-5}$. The main matched E-value, between $10^{-50}$ and $10^{-20}$, included 510 searched unique sequences, which was more than $70 \%$ of the 724 matched sequences. The remainder of the unique sequences (39.4\%) had no meaningful matches $\left(\mathrm{E}>10^{-5}\right)$.

Given that Drosophila is the most thoroughly annotated insect genome [11], we compared $S$. frugiperda unique sequences with Drosophila genes using BLASTX. A total of 677 sequences had hits with Drosophila genes at $\mathrm{E}<10^{-5}$, that is, $56.7 \%$ of the 1,195 unique sequences (Table 2 ). A subset of 53 unique sequences (4.4\%) matched to Drosophila genes with the cut-off equal to $\mathrm{E}<10^{-100}$. A total of 274 sequences $(22.9 \%)$ had matches with Drosophila genes between $10^{-100}$ and $10^{-50}, 224$ sequences (18.7\%) had matches between E-values of $10^{-50}$ and $10^{-20}$, and 116 sequences $(9.7 \%)$ had matches between E-values of $10^{-20}$ and $10^{-5}$ (Table 2 ).

We compared our unique sequences from the SF-21 cell line with ESTs obtained from another $S$. frugiperda-derived cell line, Sf9 [12], using BLASTN [13]. A total of 419 sequences (35\%) matched the ESTs from Sf9 cells with an E-value equal to 0 (Table 5). A total of 241 sequences (20.2\%) were similar but not exact matches with ESTs from Sf9 with E-values $>0$ and $<10^{-5}$. In addition, almost half of our ESTs (535 sequences or $44.8 \%$ ) had no significant match with the Sf9 ESTs. Therefore, 776 of our sequences $(65 \%)$ were not previously reported in the Sf9 EST project.

We also compared our sequences with about 4,000 S. frugiperda midgut-specific ESTs available in NCBI dbEST database using BLASTN. Only 88 sequences $(7.36 \%)$ matched with E-value equal to 0 (Table 5). The remaining sequences matched midgut ESTs to different extents: 19 sequences $(1.59 \%)$ had E-values between 0 and 10-150, 20 sequences $\left(1.67 \%\right.$ ) had E-values of $10^{-150}$ and $10^{-100}, 24$ sequences $(2.01 \%)$ had E-values of $10^{-100}$ and $10^{-50}, 31$ sequences $(2.59 \%)$ had E-values of $10^{-50}$ and $10^{-20}$, and 76 sequences $(6.36 \%)$ had E-values of $10^{-20}$ and $10^{-5}$. A total of 937 sequences $(78.4 \%)$ had no hits with the available midgut ESTs.
In addition, we compared our unique sequences with those of the silkworm B. mori. We used BLASTN to search the all B. mori EST sequences available using a BLAST search site [14], given that the genome sequence is not fully annotated. A total of 492 from the 1,195 unique sequences $(41.17 \%)$ had hits with silkworm sequences at $\mathrm{E}<10^{-5}$ (Table 6). Of these, 133 unique ESTs (27\% of the 492 sequences) had E-values between $10^{-100}$ and $10^{-50}$. A total of 703 sequences $(58.8 \%$ ) had no matches with silkworm sequences.

Conserved S. frugiperda and Drosophila gene sequences We found 11 highly conserved sequences between $S$. frugiperda and Drosophila genes based on BLASTX analyses. All of the 11 sequences were from contigs, with one, contig 134, having an E-value of 10-154. Six sequences had matches with their homologous Drosophila genes at an Evalue of 0 .

We chose contig 134 for phylogenetic analysis given it was the most conserved sequence between Spodoptera and Drosophila. The sequences from heat shock 70 cognate 4 proteins were aligned with CLUSTALW and only similar sequences with complete coding sequences (CDS) were included in the alignment as described in Methods. Alignments of the heat shock protein 70 cognate 4 (contig 134) with similar ones in the Class Insecta (Fig. 2) showed that the heat shock protein 70 cognate 4 of $S$. frugiperda formed a single clade with Trichoplusia ni, Manduca sexta, Bombyx mori, and Lonomia oblique, as expected since all these organisms belong to the order Lepidoptera. This clade shares a common ancestor with members of other orders, Diptera, Orthoptera, and Hymenoptera, and with insects in other clades (e.g., Ceratitis capitata,Chironomus tentans, Drosophila melanogaster, Anopheles gambiae, Locusta migratoria, and Cotesia rubecula).

\section{Functional classification of S. frugiperda ESTs}

Gene Ontology (GO) has been widely used to characterize gene function annotation and classification [15]. GO describes gene function using controlled vocabulary and hierarchy including molecular function, biological processes, and cellular communication. In this report, we used well annotated GO information of Drosophila melanogaster to interpret the gene function of our ESTs. Each unique sequence from $S$. frugiperda was assigned the same gene function of the best BLASTX hit $\left(\mathrm{E} \leq 10^{-5}\right)$ with Drosophila sequences based on the annotated GO of Drosophila [15]. This method has been successfully used to annotate bee brain EST function [16].

The major GO categories for the unique sequences included those outlining gene molecular function (Additional file 1-Table 7), biological processes (Additional file 2- Table 8), and cellular components (Additional file 3- 
Table I: Most abundantly represented transcripts in the Spodoptera frugiperda cDNA library.

\begin{tabular}{|c|c|c|c|c|c|c|c|}
\hline Contig & ESTs & Gl\# & Bit score & E-value & Identities & Gene descriptions & Organism \\
\hline Contig 190 & 13 & 40363707 & 238 & $|e-6|$ & $129 / 215$ & cytochrome oxidase II & Glyphodes bicolor \\
\hline Contig 98 & 13 & 18314310 & 241 & $5 e-96$ & $122 / 159$ & Cytochrome c oxidase subunit 3 & Ostrinia furnacalis \\
\hline Contig II6 & 13 & 16566722 & 458 & e- 128 & $229 / 244$ & ribosomal protein $\mathrm{S} 3 \mathrm{~A}$ & S. frugiperda \\
\hline Contig I46 & 13 & 18253045 & 138 & $5 e-32$ & $76 / 112$ & 60 s acidic ribosomal protein P2 & S. frugiperda \\
\hline Contig 6I & 14 & 7302066 & 249 & $6 e-65$ & $129 / 243$ & CGII522-PB, isoform B & D. melanogaster \\
\hline Contig 225 & 14 & 18253043 & 147 & le-34 & $78 / 111$ & 60 s acidic ribosomal protein PI & S. frugiperda \\
\hline Contig 70 & 15 & 54609281 & 452 & e- 126 & $233 / 307$ & ribosomal protein SA & B. mori \\
\hline Contig 139 & 17 & 27260896 & 411 & e-113 & $204 / 218$ & ribosomal protein S2 & S. frugiperda \\
\hline Contig 160 & 17 & 22094837 & 389 & e- 106 & 199/283 & Cytochrome b & Samia cynthia ricini \\
\hline Contig 23 & 18 & $|825304|$ & 550 & e-155 & $283 / 315$ & 60 Saccadic ribosomal protein $\mathrm{PO}$ & S. frugiperda \\
\hline Contig 134 & 21 & $|258526|$ & 1119 & 0 & $568 / 608$ & Heat shock 70 kDa cognate 4 & Manduca sexta \\
\hline Contig 239 & 30 & 39752635 & 480 & 0 & $234 / 241$ & elongation factor-I alpha F2 & D. melanogaster \\
\hline Contig I4I & 46 & 1438928 & 685 & 0 & $364 / 504$ & Cytochrome oxidase subunit I & Feltia jaculifera \\
\hline Contig 19 & 63 & 552886 & 226 & e-III & $102 / 104$ & ND-I protein gene & S. frugiperda \\
\hline
\end{tabular}

Table 9). The highest final child GO term for molecular function was the hydrogen transporting two-sector ATPase in the nucleotide binding category. The highest final GO term for biological processes was protein biosynthesis, under the protein metabolism and biosynthesis categories, which had 84 unique sequences accounting for $7 \%$ of the total unique sequences matched in this category. The largest number for a final GO term in cellular components was cytosolic large ribosomal subunit under both the ribosome and cytosol categories. Seventy-eight unique sequences belonged to this GO term, which accounted for $6.5 \%$ of the total unique sequences annotated for cellular components.

We found 13 unique sequences (1.1\%) showing significant similarity with Drosophila signal transduction factors (Table 3). Among these, 6 sequences belonged to the receptor binding category and the remaining 7 sequences belonged to receptor and receptor signaling proteins.

Based on GO, we also found one sequence for an apoptosis-related gene, pyes2-ct_017_g10.p1ca, which showed similarity to the Dros ophila Aac11 gene. Two additional sequences, pb42ad-1_001_f09.pb42 primer and pyes2ct_010_g11.p1ca, showed significant similarity to Drosophila Gnbp3, a gene involved in defense and immunity.

\section{Pathway analysis based on the Kyoto Encyclopedia of Genes and Genomes (KEGG) classification}

KEGG has been widely used for pathway mapping [17]. Enzyme commission (EC) numbers were used to judge which sequences pertained to a specific pathway. Twentynine unique sequences including 8 contigs and 21 singlets accounted for $2.4 \%$ of unique sequences and matched enzymes with an EC number. Within these 29 sequences, 11 unique sequences ( $1 \%$ of total) containing 4 contigs and 7 singlets were mapped to KEGG biochemical pathways (Table 4). Genes involved in amino acid metabolism had the highest mapping sequences (5) and 6 mappings. Genes involved in carbohydrate metabolism included 5 sequences and 8 mappings. Other genes included those involved in nucleotide metabolism ( 2 sequences), translation ( 2 sequences), energy metabolism (1 sequence), lipid metabolism (1 sequence), and metabolism of other

Table 2: Comparative analysis of Spodoptera ESTs to Drosophila' and other sequences.

\begin{tabular}{|c|c|c|c|c|c|c|c|c|c|c|c|c|}
\hline \multirow[b]{3}{*}{ Homology } & \multicolumn{6}{|c|}{ All matches } & \multicolumn{6}{|c|}{ Drosophila } \\
\hline & \multicolumn{2}{|c|}{ Contig } & \multicolumn{2}{|c|}{ Singlets } & \multicolumn{2}{|c|}{ Total } & \multicolumn{2}{|c|}{ Contig } & \multicolumn{2}{|c|}{ Singlets } & \multicolumn{2}{|c|}{ Total } \\
\hline & $\mathbf{N}$ & $\%$ & $\mathbf{N}$ & $\%$ & $\mathbf{N}$ & $\%$ & $\mathbf{N}$ & $\%$ & $\mathbf{N}$ & $\%$ & $\mathbf{N}$ & $\%$ \\
\hline$E \leq 10-150$ & 11 & 5 & 0 & 0 & 11 & 2 & 10 & 5 & 0 & 0 & 10 & 1 \\
\hline$E \leq 10-100$ & 30 & 15 & 23 & 4 & 53 & 7 & 30 & 16 & 23 & 5 & 53 & 8 \\
\hline$E \leq 10-50$ & 92 & 45 & 191 & 37 & 283 & 39 & 88 & 46 & 186 & 38 & 274 & 40 \\
\hline$E \leq 10-20$ & 48 & 24 & 189 & 36 & 237 & 33 & 45 & 24 & 179 & 37 & 224 & 33 \\
\hline$E \leq 10^{-5}$ & 22 & 11 & 118 & 23 & 140 & 20 & 17 & 9 & 99 & 20 & 116 & 17 \\
\hline Total matched & 203 & 91 & 521 & 55 & 724 & 61 & 190 & 85 & 487 & 50 & 677 & 57 \\
\hline No match & 41 & 9 & 430 & 45 & 471 & 39 & 54 & 22 & 484 & 50 & 518 & 43 \\
\hline Total & 244 & 100 & 951 & 100 & 1195 & 100 & 244 & 100 & 951 & 100 & 1195 & 100 \\
\hline
\end{tabular}

' [II] 
Table 3: Signal transduction sequences of Spodoptera frugiperda compared with Drosophila genes.

\begin{tabular}{|c|c|c|c|c|c|c|c|}
\hline S. frugiperda sequences & $\begin{array}{l}\text { Flybase } \\
\text { number }\end{array}$ & $\begin{array}{l}\text { Hit } \\
\text { length }\end{array}$ & $\begin{array}{c}\text { Bit } \\
\text { score }\end{array}$ & E-value & Identities & $\begin{array}{l}\text { Drosophila } \\
\text { gene }\end{array}$ & Gene description \\
\hline pyes2-ct_019_b03.plca & FBgn003954I & 836 & 275 & $1.00 \mathrm{e}-74$ & $138 / 266$ & $C g / 2876$ & Signal transduction activity \\
\hline Pyes2-ct_006_fl2.plca & FBgn003577I & 753 & 360 & e- 104 & $|64 / 23|$ & Cg8583 & Signal recognition particle binding \\
\hline pyes2-ct_02I_al2.plca & FBgn0027363 & 689 & 84 & $1.00 \mathrm{e}-17$ & $35 / 59$ & Stam & Signal transducing adaptor molecule \\
\hline Contig 14 & FBgn0003963 & 1191 & 139 & $2.00 e-33$ & $86 / 223$ & ush & Involved in torso signaling pathway \\
\hline Pyes2-ct_005_g0l.plca & FBgn003577I & 753 & 222 & $1.00 e-58$ & $104 / 149$ & $\operatorname{cg} 8583$ & $\begin{array}{l}\text { Involved in signal recognition particle } \\
\text { complex }\end{array}$ \\
\hline Pyes2-ct_006_f09.plca & FBgn0037277 & 2228 & 314 & $3.00 e-86$ & $152 / 242$ & $\mathrm{Cg} / 7735$ & $\begin{array}{l}\text { Ligand-dependent nuclear receptor } \\
\text { interactor activity }\end{array}$ \\
\hline pyes2-ct_028_g06.plca & FBgn0020618 & 318 & 199 & $|.00 e-5|$ & $96 / 107$ & Rack I & Receptor of activated protein kinase C I \\
\hline Contig 140 & FBgn0020618 & 318 & 578 & e- 165 & $274 / 319$ & Rack I & Receptor of activated protein kinase C I \\
\hline pyes2-ct_030_g06.plca & FBgn0004569 & 444 & 67 & $4.00 \mathrm{e}-12$ & $29 / 43$ & argos & Receptor antagonist activity \\
\hline p42ad_2_00I_b07.plcb.exp & FBgn0037II3 & 1258 & 133 & $3.00 \mathrm{e}-32$ & $71 / 131$ & $\operatorname{cg} 33291$ & Putative protein binding \\
\hline pyes2-ct_003_el2.plca & FBgn00I 3984 & 2144 & 73 & $1.00 \mathrm{e}-13$ & $74 / 313$ & $\ln R$ & Insulin like receptor \\
\hline Contig 220 & FBgn003। 547 & 406 & 125 & $3.00 e-43$ & $65 / 191$ & CG32 I 2 & $\begin{array}{l}\text { Scavenger receptor activity involved in } \\
\text { defense response }\end{array}$ \\
\hline Contig 226 & FBgn0037357 & 773 & 102 & $7.00 \mathrm{e}-25$ & $52 / 62$ & $\sec 23$ & Putative GTPase activator activity \\
\hline
\end{tabular}

amino acids ( 1 sequence). Contigs 35 and 97 each had 4 mappings to different pathways under a different metabolism category, which was the highest number for a single sequence. Contig 120, contig 152, pyes2ct_008_e11.p1ca, and pyes2-ct_012_c04.p1ca mapped to two pathways. The remaining pathway-assigned sequences only mapped to one pathway.

\section{EST database}

To efficiently manage and retrieve information in the ESTs analyzed in this project, we developed an EST model database (ESTMD version 1) [9]. The EST model database is a highly efficient, web-accessible, user-friendly relational database. It provides several tools to search raw, cleaned, and assembled EST sequences, genes and GO, as well as pathway information. The user may input and submit keywords or IDs to the server using the web interface. ESTs and annotated function data are in the relational database

Table 4: KEGG pathway mapping for Spodoptera frugiperda unique sequences.

\begin{tabular}{|c|c|c|c|}
\hline KEGG pathway & Sequence ID & $\begin{array}{l}\text { Number of } \\
\text { sequences }\end{array}$ & $\begin{array}{c}\text { Percentage of } \\
\text { total }\end{array}$ \\
\hline Carbohydrate metabolism & & 4 & 36 \\
\hline Glycolysis/gluconeogenesis & Contig 35, contig 97, pyes2-ct_008_el I.pl ca & 3 & 27 \\
\hline Fructose and mannose metabolism & pyes2-ct_027_b04.plca & 1 & 9 \\
\hline Pyruvate metabolism & Contig 35 , contig 97 & 2 & 18 \\
\hline Propanoate metabolism & Contig 35 & 1 & 9 \\
\hline Starch and sucrose metabolism & pyes2-ct_0I0_glI.plca & 1 & 9 \\
\hline Energy metabolism & & I & 9 \\
\hline Carbon fixation & Contig 97 & 1 & 9 \\
\hline Lipid metabolism & & 1 & 9 \\
\hline Sphingoglycolipid metabolism & Pyes2-ct_010_a06.plca & 1 & 9 \\
\hline Nucleotide metabolism & & 2 & 18 \\
\hline Purine metabolism & Contig 97, contig 120 & 2 & 18 \\
\hline Amino acid metabolism & & 5 & 45 \\
\hline Alanine and aspartate metabolism & Contig 120 & 1 & 9 \\
\hline Arginine and proline metabolism & pyes2-ct_026_hI I.plca & 1 & 9 \\
\hline Glycine, serine and threonine metabolism & pyes2-ct_012_c04.plca & 1 & 9 \\
\hline Cysteine metabolism & Contig 35 & 1 & 9 \\
\hline Phenylalanine, tyrosine and tryptophan biosynthesis & Contig 152, pyes2-ct_008_ell.plca & 2 & 18 \\
\hline Metabolism of other amino acids & & I & 9 \\
\hline Selenoamino acid metabolism & pyes2-ct_02I_fl0.plca & I & 9 \\
\hline Translation & & 2 & 18 \\
\hline Aminoacyl-tRNA biosynthesis & Contig 152, pyes2-ct_012_c04.pl ca & 2 & 18 \\
\hline
\end{tabular}


Table 5: Comparative analysis of Spodoptera frugiperda SF-2 I-derived ESTs with Sf9- and midgut-derived ESTs'.

\begin{tabular}{|c|c|c|c|c|c|c|c|c|c|c|c|c|}
\hline \multirow[b]{3}{*}{ Homology } & \multicolumn{6}{|c|}{ S. frugiperda Sf9ESTs } & \multicolumn{6}{|c|}{ S. frugiperda midgutESTs } \\
\hline & \multicolumn{2}{|c|}{ Contig } & \multicolumn{2}{|c|}{ Singlets } & \multicolumn{2}{|c|}{ Total } & \multicolumn{2}{|c|}{ Contig } & \multicolumn{2}{|c|}{ Singlets } & \multicolumn{2}{|c|}{ Total } \\
\hline & $\mathbf{N}$ & $\%$ & $\mathbf{N}$ & $\%$ & $\mathbf{N}$ & $\%$ & $\mathbf{N}$ & $\%$ & $\mathbf{N}$ & $\%$ & $\mathbf{N}$ & $\%$ \\
\hline$E \leq 0$ & 243 & 99.59 & 176 & $|8.5|$ & 419 & 35.06 & 7 & 2.87 & 81 & 8.52 & 88 & 7.36 \\
\hline $0<E \leq 10^{-150}$ & 0 & 0.00 & 37 & 3.89 & 37 & 3.10 & 2 & 0.82 & 17 & 1.79 & 19 & 1.59 \\
\hline $10^{-150}<\mathrm{E} \leq 10^{-100}$ & 0 & 0.00 & 47 & 4.94 & 47 & 3.93 & 4 & 1.64 & 16 & 1.68 & 20 & 1.67 \\
\hline $10^{-100}<\mathrm{E} \leq 10^{-50}$ & 0 & 0.00 & 58 & 6.10 & 58 & 4.85 & 0 & 0.00 & 24 & 2.52 & 24 & 2.01 \\
\hline $10^{-50}<\mathrm{E} \leq 10^{-20}$ & 0 & 0.00 & 49 & 5.15 & 49 & 4.10 & I & 0.41 & 30 & 3.15 & 31 & 2.59 \\
\hline $10^{-20}<\mathrm{E} \leq 10^{-5}$ & 0 & 0.00 & 50 & 5.26 & 50 & 4.18 & 4 & 1.64 & 72 & 7.57 & 76 & 6.36 \\
\hline Total matched & 243 & 99.59 & 417 & 43.85 & 660 & 55.23 & 18 & 7.38 & 240 & 25.24 & 258 & 21.59 \\
\hline No match & I & 0.41 & 534 & 56.15 & 535 & 44.77 & 226 & 92.62 & 711 & 74.76 & 937 & 78.41 \\
\hline Total & 244 & 100 & 951 & 100 & 1195 & 100 & 244 & 100 & 951 & 100 & 1195 & 100 \\
\hline
\end{tabular}

I SF-2I ESTs, this report; Sf9 ESTs [12]; midgut ESTs (NCBI dbEST)

and results are sent back to the user in proper formats in response to a query. It also provides a clear contig view and BLAST searches, data submission, and download pages. In both the GO and KEGG pathway search pages, ESTMD allows users to search GO and pathways not only by single gene name, symbol, or ID, but also by using a file that contains a batch of sequence IDs or FlyBase IDs. All the sequence function classification based on GO and KEGG pathway in this study was done using ESTMD. The file search provision in ESTMD allows users to obtain information regarding the possible function of many ESTs or genes at one time instead of searching them individually (Fig. 3).

\section{Discussion}

Single-pass sequencing was performed on 3,365 cDNA clones derived from two SF-21 cDNA libraries. Using this sequence data, we have established an EST database comprised of 1,195 unique sequences from the SF-21 cell line, derived from the lepidopteran insect $S$. frugiperda. A total of 667 unique sequences $(57 \%)$ had homology to sequences found in Drosophila. These sequences will be useful for comparative genomics within and outside the Lepidoptera, establishing microarrays, and as probes to either clone or down-regulate genes of interest by RNA interference in order to perform studies related to Spodoptera, other closely related Lepidoptera, or their pathogens.

This is one of two published annotated EST studies available for $S$. frugiperda. A prior report included a similar scale project as that reported here with 5,937 ESTs of which 1,855 were unique sequences obtained from a clonally-derived cell line of SF-21, Sf9 [12]. The majority of unique sequences in the previous study consisted of the highly abundant ribosomal protein genes and these were found to have low codon usage bias [12]. Our data provides 776 novel $S$. frugiperda sequences. A small percentage of our sequences $(20.2 \%)$ had similarities, whether these reflect cell line specific differences is not clear at this time. Together these two studies and other available $S$. frugiperda ESTs constitute seminal work on the genome sequence of $S$. frugiperda. The sequences reported in this

Table 6: Comparative analysis of Spodoptera frugiperda SF-2I ESTs with silkworm ESTs.

\begin{tabular}{|c|c|c|c|c|c|c|}
\hline \multirow[b]{3}{*}{ Homology } & \multicolumn{6}{|c|}{ Bombyx mori ESTs } \\
\hline & \multicolumn{2}{|c|}{ Contig } & \multicolumn{2}{|c|}{ Singlets } & \multicolumn{2}{|c|}{ Total } \\
\hline & $\mathbf{N}$ & $\%$ & $\mathbf{N}$ & $\%$ & $\mathbf{N}$ & $\%$ \\
\hline$E \leq 0$ & 53 & 21.72 & 7 & 0.74 & 60 & 5.02 \\
\hline $0<\mathrm{E} \leq 10^{-150}$ & 17 & 6.97 & 12 & 1.26 & 29 & 2.43 \\
\hline $10^{-150}<\mathrm{E} \leq 10^{-100}$ & 51 & 20.90 & 60 & 6.31 & 111 & 9.29 \\
\hline $10^{-100}<\mathrm{E} \leq 10^{-50}$ & 44 & 18.03 & 89 & 9.36 & 133 & 11.13 \\
\hline $10^{-50}<\mathrm{E} \leq 10^{-20}$ & 13 & 5.33 & 79 & 8.31 & 92 & 7.70 \\
\hline $10^{-20}<\mathrm{E} \leq 10^{-5}$ & 13 & 5.33 & 54 & 5.68 & 67 & 5.61 \\
\hline Total matched & 191 & 78.28 & 301 & 31.65 & 492 & 41.17 \\
\hline No match & 53 & 21.72 & 650 & 68.35 & 703 & 58.83 \\
\hline Total & 244 & 100 & 951 & 100 & 1195 & 100 \\
\hline
\end{tabular}

I SF-2I ESTs, this report; Sf9 ESTs [12]; midgut ESTs (NCBI dbEST) 

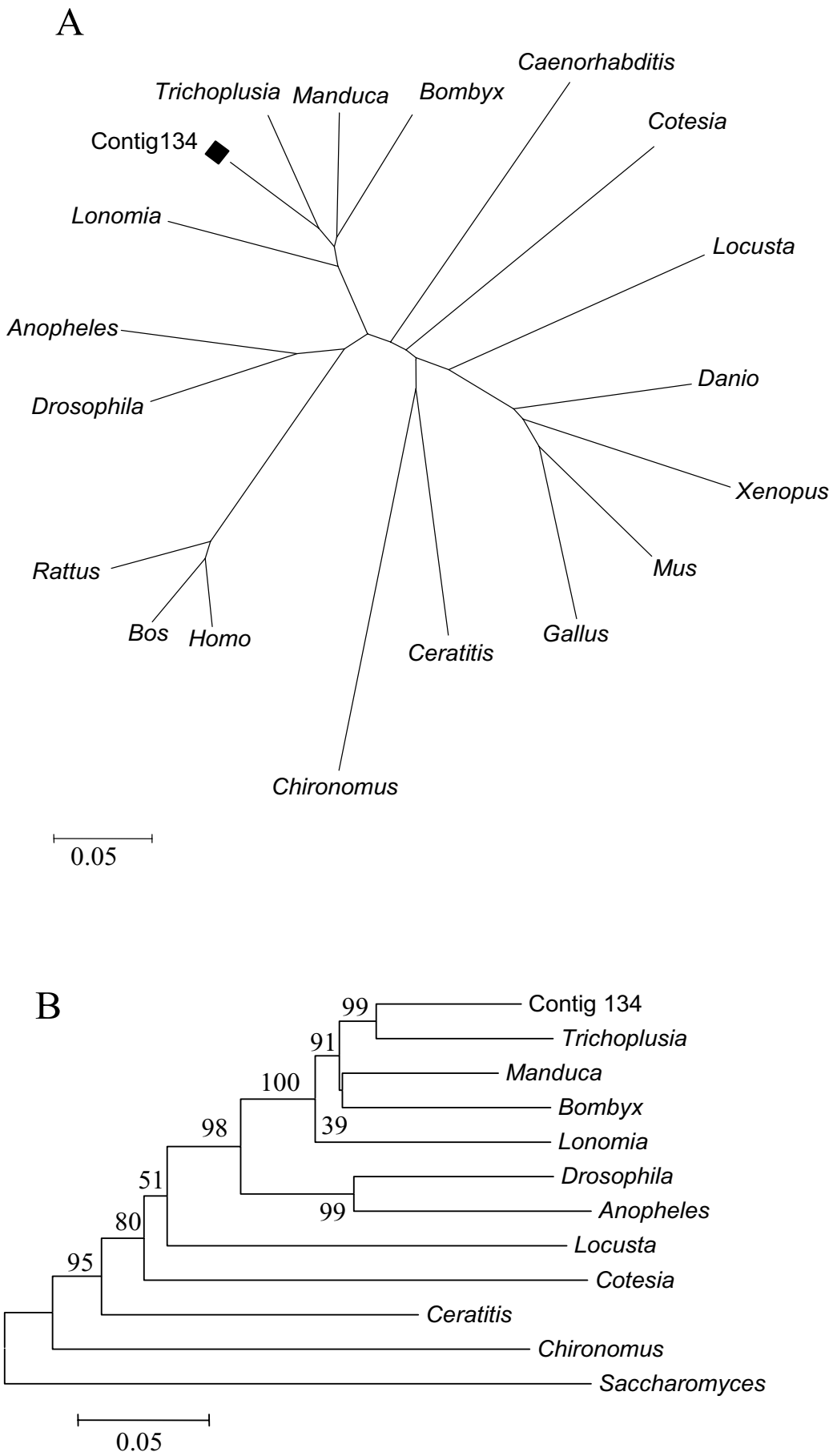

Figure 2

Phylogenetic analysis with neighbor-joining tree. A. The heat shock proteins of 10 insects and 8 other organisms (Cotesia rubecula, Ceratitis capitata, Chironomus tentans, Manduca sexta, Locusta migratoria, Drosophila melanogaster, Anopheles gambiae, Lonomia oblique, Bombyx mori, Trichoplusia ni, Bos taurus, Gallus gallus, Rattus norvegicus, Danio rerio, Xenopus laevis, Caenorhabditis elegans, Mus musculus, Homo sapiens) along with Contig 134 (heat shock 70 cognate 4 protein) are presented in unrooted phylogenetic analyses. B. Phylogenetic tree showing heat shock proteins with Saccharomyces cerevisiae as the outgroup. The bootstrap values (percentages) are indicated at the corresponding node. 
study have been made available for incorporation into Spodobase [18].

Many insects within the Lepidoptera, including the fall armyworm $S$. frugiperda (family Noctuidae), are pests that cause significant annual damage to a number of field crops and tree foliage worldwide. Deciphering their genomic sequences will aid in developing improved pest control agents, such as baculoviruses and polydnaviruses/ parasitic wasps. Although these pathogens are being used or sought as biological control agents, there remains ample room for improvement of their entomopathogenic properties.

Finally, molecular tools have been used in the study of Lepidoptera or have been derived from Lepidoptera. The transposable element piggyBac was discovered in the lepidopteran T. ni (cabbage looper) and has been used to create somatic and germline transformations in a number of organisms including crickets, butterflies, Plasmodium falciparum, and more recently, mice [19-22]. Lepidoptera are also amenable to down-regulation of genes by RNA interference and transgenic techniques [23]. Thus, knowledge of the genomics of Lepidoptera will aid in their manipulation or use as molecular tools.

\section{Conclusion}

We have established an EST database from the $S$. frugiperda-derived cell line SF-21, containing 1,195 unique sequences. Lepidoptera are among the most diverse insects and as such, sequences and EST databases from various genomes will be instrumental in assessing speciesspecific genes, phylogeny, and parallels within species of the same order. In addition, comparative analyses with available genomes of other insects including $A$. mellifera, D. melanogaster, A. gambiae, Ae. aegypti, and T. castaneum will yield additional insights since these include members of distinct orders (Hymenoptera, Diptera, and Coleoptera), providing a more accurate picture of the conserved pathways and the order-specific gene elements in the Insecta.

\section{Methods \\ cDNA library construction}

Two independently constructed cDNA libraries were used for sequencing. For both libraries, mRNA isolated from log phase SF-21 cells was used for CDNA synthesis, and the libraries were directionally cloned into plasmid vectors. One library was custom made by Clontech using the plasmid vector pB42AD. The pB42AD library had a titer of 3.6 $\times 10^{13}$ colony forming units per $\mathrm{ml}$. The second library was constructed using the SuperScript ${ }^{\mathrm{TM}}$ Plasmid System (Invitrogen) and the plasmid vector pYES2/CT (Invitrogen) that had been modified by addition of a Sal I linker at the Bam HI site. The pYES2/CT library had a titer of 1.2 $\times 10^{12}$ colony forming units per $\mathrm{ml}$. The average insert size for both libraries was $1.5 \mathrm{kbp}$.

\section{EST sequencing}

Initially, approximately 200 randomly selected clones from each library were subjected to single-pass sequencing using 5 ' vector primers. DNA sequencing was performed by MWG Biotech (High Point, NC). Although both libraries yielded acceptable sequence quality, the pYES2/CT library appeared to yield slightly longer sequences; consequently, the remainder of the sequencing was performed using clones from the pYES2/CT library.

\section{Sequence processing}

Sequence information was stored in chromatograph trace files, and Phred [24] was used to perform Base-calling [24]. Flanking vector and adaptor sequences were trimmed using Cross-match [25] and Lucy [26], while low quality bases (quality score < 20) were cleaned at both sequence ends by using our custom program. RepeatMasker [27] was used to mask repeated sequences, and the masked sequences were further screened to remove contaminating sequences from bacteria and viruses using BLASTN [13]. High quality ESTs were assembled using CAP 3 [7] and verified with Phrap [8], which perform similar tasks. After assembly, Consed [24] was used to assess contig quality, and assembled ESTs were chosen for further analysis. Contigs flagged for possible miss-assembly were manually edited in Consed and potential chimeric ESTs or other suspect ESTs were removed from the pool of traces.

\section{Sequence annotation}

High quality assembled ESTs were annotated using BLASTX through NCBI and our local BLAST server. We searched several databases including the NCBI nonredundant and Drosophila protein databases. The BLAST results were automatically extracted and transferred into a relational database. The sequences reported in this study (2,367 ESTs) have been deposited in GenBank under accession numbers [GenBank: DY792773 to DY795139].

\section{Functional classification}

Functional classification of unique sequences from $S$. frugiperda was based on GO [15]. Unique sequences, including contigs and non-overlapping singlets, were used to search Drosophila predicted protein databases using BLASTX. The Drosophila genes corresponding to the best hits at a threshold of E-value $\leq 10^{-5}$ with known GO term were assigned to the query "Spodoptera sequences". All the matched GO information was stored in our local MySQL database. 


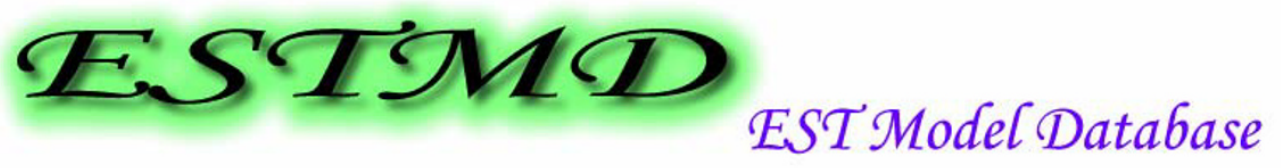

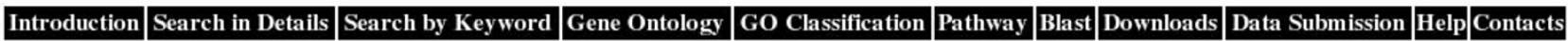

GO Sequence Search Result

\begin{tabular}{|l|l|l|l|l|l|l|l|}
\hline SequenceID & \multicolumn{1}{|c|}{ GO_ID } & \multicolumn{1}{|c|}{ GO_Term } & Type & & \multicolumn{1}{|c|}{ HitID } & Gene_Symbol \\
\hline Contig1 & GO:0003735 & structural constituent of ribosome & molecular_function & FBgn0004413 & RpS26 \\
\hline Contig1 & GO:0006412 & protein biosynthesis & biological_process & FBgn0004413 & RpS26 \\
\hline Contig1 & GO:0005843 & cytosolic small ribosomal subunit (sensu Eukarya) & cellular_component & FBgn0004413 & RpS26 \\
\hline Contig1 & GO:0005840 & ribosome & cellular_component & FBgn0004413 & RpS26 \\
\hline Contig10 & GO:0006412 & protein biosynthesis & biological_process & FBgn0011272 & RpL13 \\
\hline Contig10 & GO:0005842 & cytosolic large ribosomal subunit (sensu Eukarya) & cellular_component & FBgn0011272 & RpL13 \\
\hline Contig10 & GO:0005840 & ribosome & cellular_component & FBgn0011272 & RpL13 \\
\hline Contig10 & GO:0003735 & structural constituent of ribosome & molecular_function & FBgn0011272 & RpL13 \\
\hline Contig106 & GO:0004772 & sterol O-acyltransferase & molecular_function & FBgn0031327 & CG5397 \\
\hline Contig35 & GO:0006096 & glycolysis & biological_process & FBgn0001258 & ImpL3 \\
\hline Contig35 & GO:0004459 & L-lactate dehydrogenase & molecular_function & FBgn0001258 & ImpL3 \\
\hline
\end{tabular}

Figure 3

Gene Ontology search results. The search results used a sequence file by choosing all three ontologies.

\section{Pathway assignments}

Pathway assignments were carried out according to KEGG mapping [17]. EC numbers [28] were assigned to unique sequences that had BLASTX scores with a cut off value of $\mathrm{E}=10^{-5}$ or less upon searching SWIR protein databases. The sequences were mapped to KEGG biochemical pathways according to the EC distribution in the pathway database.

\section{Phylogenetic analysis}

Proteins were aligned with CLUSTALW using only CDS in the alignment. The alignment was then used to generate phylogenetic trees by the Neighbour-Joining method using the MEGA version 2.1 program. The bootstrap values for the nodes were determined by analyzing 500 bootstrap replicate data sets to estimate the strength of the groupings.

\section{Database implementation}

A web-based interface of the database was created using HTML and JavaScript to evaluate the validation of the input on the client side and to reduce the burden on the server side. Apache 2.0 was used as the http web server, while Tomcat 4.1 was the servlet container. Both of these programs were developed and maintained on UNIX, Linux, and Windows NT, ensuring that ESTMD was transplantable and platform-independent. ESTMD is currently hosted on Red Hat 9, and it can be implemented in
MySQL 4.0 or higher version. The main tables were on clones, ESTs, uniSequence, uniHit, FlyBase and FlyBaseDetails. The server-side programs were implemented by Java technologies. Servlet and JavaServer Pages were used to communicate between users and databases and to implement a query. XML and XSLT technologies were used to describe, generate, and express GO trees.

\section{Authors' contributions}

ALP and RJC contributed to the conception and design of the study, coordinated the study, and were involved in drafting the manuscript. YDeng participated in data analysis and drafting of the manuscript. YDong and VT were involved in data analysis. All authors read and approved the final manuscript.

\section{Additional material}

\section{Additional file 1- Table 7}

Table 7. Distribution of molecular functional categories based on gene ontology for Spodoptera frugiperda unique sequences Click here for file

[http://www.biomedcentral.com/content/supplementary/14712164-7-264-S1.pdf] 


\section{Additional file 2- Table 8}

Table 8. Distribution of biological processes based on gene ontology for Spodoptera frugiperda unique sequences

Click here for file

[http://www.biomedcentral.com/content/supplementary/1471-

2164-7-264-S2.pdf]

\section{Additional file 3- Table 9}

Table 9. Distribution of cellular component categories based on gene ontology for Spodoptera frugiperda unique sequences

Click here for file

[http://www.biomedcentral.com/content/supplementary/1471-

2164-7-264-S3.pdf]

\section{Acknowledgements}

We thank the late Lois K. Miller and Casey W. Wright for providing the cDNA libraries, Yonghua Li for help with the database, Vijayaraj Nagarajan for help with phylogenetic analysis, and Kuan Yang for help with sequence comparisons.

This work was supported in part by the NIH National Center for Research Resources awards P20 RR I6443, P20 RR 107686, P20 RRI6475, and P20RR016476. Y. Deng was also supported by the Dean's Research Initiative award of the University of Southern Mississippi. This is contribution number 06-273-J from the Kansas Agricultural Experiment Station.

\section{References}

I. Adams MD, Celniker SE, Holt RA, Evans CA, Gocayne JD, Amanatides PG, Scherer SE, Li PW, Hoskins RA, Galle RF, George RA, Lewis SE, S. R, Ashburner M, Henderson SN, Sutton GG, Wortman JR, Yandell MD, Zhang Q, Chen LX, Brandon RC, Rogers YHC, Blazej RG, Champe M, Pfiffer BD, Wan KH, Doyle C, Baxter EG, Helt G, Nelson CR, Miklos GLG, Abril JF, Agbayani A, An HJ, Andrews-Pfannkoch C, Baldwin D, Ballew RM, Basu A, Baxendale J, Bayraktaroglu L, Beasley $\mathrm{EM}$, Beeson KY, Benos PV, Bernam BP, Bhandari D, Bolshakov S, Borkova D, Botchan MR, Bouck J, Brokstein P, Brottier P, Burtis KC, Busam DA, Butler H, Cadieu E, Center A, Candra I, Cherry JM, Cawley S, Dahlke C, Daenport LB, Davies P, de Pablos B, Delcher A, Deng Z, Days AD, Dew I, Dietz SM, Dodson K, Doup LE, Downes M, Dugan-Rocha S, Dunkov BC, Dunn P, Durbin KJ, Evangelista CC, Ferraz C, Ferriera S, Fleischmann W, Fosler C, Gabrielian AE, Garg NS, Gelbart WM, Glasser K, Glodek A, Gong F, Gorrell JH, Gu Z, Guan P, Harris M, Harris NL, Harvey D, Heiman TJ, Hernandez JR, Houck J, Hostin D, Houston KA, Howland TJ, Wei MH, lbegwam C, Jalali M Kalush F, Karpen GH, Ke Z, Kennison JA, Ketchum KA, Kimmel BE, Kodira CD, Kraft C, Kravitz S, Kulp D, Lai Z, Lasko P, Lei Y, Levitsky AA, Li J, Li Z, Liang Y, Lin X, Liu X, Mattei B, Mclntosh TC, McLeod MP, McPherson D, Merkulov G, Milshina NV, Mobarry C, Morris ], Moshrefi A, Mount SM, Moy M, Murphy B, Murphy L, Muzny DM, Nelson DL, Nelson DR, Nelson KA, Nixon K, Nusskern DR, Pacleb JM, Palazzolo M, Pittman GS, Pan S, Pollard J, Puri V, Reese MG, Reinert K, Remington K, Saunders RDC, Scheeler F, Shen H, Shue BC, SidenKiamos I, Simpson M, Skupski MP, Smith T, Spier E, Spradlling AC, Stapleton M, Strong R, Sun E, Svirskas R, Tector C, Turner R, Venter E, Wang $A H$, Wang $X$, Wang ZY, Wassarman DA, Weinstock GM, Weissenbach J, Williams SM, Woodage T, Worley KC, Wu D, Yang S, Yao A, Ye J, Yeh RF, Zaveri JS, Zhang M, Zhang G, Zhao Q, Zheng L, Zheng XH, Zhong FN, Zhong W, Zhou X, Zhu S, Zhu X, Smith HO, Gibbs RA, Myers EW, Runbin GM, Venter JC: The genome sequence of Drosophila melanogaster. Science 2000, 287:2185-2195.

2. Biology analysis group: Xia Q, Zhou Z, Lu C, Cheng D, Dai F, Li B, Zhao P, Zha X, Cheng T, Chai C, Pan G, Xu J, Liu C, Lin Y, Qian J, Hou Y, Wu Z, Li G, Pan M, Li C, Shen Y, Lan X, Yuan L, Li T, Xu H, Yang G, Wan Y, Zhu Y, Yu M, Shen W, Wu D, Xiang Z, Genome analysis group: Yu J, Wang J, Li R, Shi J, Li H, Li G, Su J, Wang X, Li G,
Zhang Z, Wu Q, Li J, Zhang Q, Wei N, Xu J, Sun H, Dong L, Liu D, Zhao S, Zhao X, Meng Q, Lan F, Huang X, Li Y, Fang L, Li C, Li D, Sun $Y$, Zhang Z, Yang Z, Huang Y, Xi Y, Qi Q, He D, Huang H, Zhang X, $X i$ Y, Qi R, He D, Huang H, Zhang X, Wang Z, Li W, Cao Y, Yu Y, Yu $\mathrm{H}$, Li J, Ye J, Chen H, Zhou Y, Liu B, Wang J, Ye J, Ji H, Li S, Ni P, Zhang J, Zhang Y, Zheng H, Mao B, Wang W, Ye C, Li S, Wang J, Wong GKS, Yang $\mathrm{H}$ : A draft sequence for the genome of the domesticated silkworm (Bombyx mori). Science 2004, 306: 1937-1940.

3. Holt RA, Subramanian GM, Halpern A, Sutton GG, Charlab R, Nusskem DR, Wincker P, Clark AG, Ribeiro JM, Wides R, Salzberg SL, Loftus B, Yandell M, Majoros WH, Rusch DB, Lai Z, Kraft CL, Abril JF, Anthouard V, Arensburger P, Atkinson PW, Baden H, de Berardinis $\mathrm{V}$, Baldwin $\mathrm{D}$, Benes $\mathrm{V}$, Biedler J, Blass $\mathrm{C}$, Bolanos R, Boscus $\mathrm{D}$, Barnstead M, Cai S, Center A, Chaturvedi K, Christophides GK, Chrystal MA, Clamp M, Cravchik A, Curwen V, Dana A, Delcher A, Dew I, Evans CA, Flanigan M, Grundschober-Freimoser A, Friedli L, Gu Z, Guan P, Guigo R, Hilllenmeyer ME, Hladun SL, Hogan JR, Hong YS, Hoover J, Jaillon O, Ke Z, Kodira C, Kokoza E, Koutsos A, Letunic I, Levitsky A, Liang Y, Lin JJ, Lobo NF, Lopez JR, Malek JA, McIntosh TC, Meister S, Miller J, Mobarry C, Mongin E, Murphy SD, O'Brochta DA, Pfannkoch C, Qi R, Regier MA, Remington K, Shao H, Sharakhova MV, Sitter CD, Shetty J, Smith TJ, Strong R, Sun J, Thomasova D, Ton LQ, Topalis P, Tu Z, Unger MF, Walenz B, Wang A, Wang J, Wang M, Wang X, Woodford KJ, Wortman JR, Wu M, Yao A, Zdobnov EM, Zhang H, Zhao Q, Zhao S, Zhu SC, Zhimulev I, Coluzzi M, della Torre A, Roth CW, Louis C, Kalush F, Mural RJ, Myers EW, Adams MD, Smith HO, Broder S, Gardner MJ, Fraser CM, Birney E, Bork P, Brey PT, Venter JC, Weissenbach J, Kafatos FC, Collins FH, Hoffman SL: The genome sequence of the malaria mosquito Anopheles gambiae. Science 2002, 298: I 29-149.

4. Mita K, Kasahara M, Sasaki S, Nagayasu Y, Yamada T, Kanamori H, Namiki N, Kitagawa M, Yamashita H, Yasukochi Y, Kadono-Okuda K, Kamamoto K, Ajimura M, Ravikumar G, Shimomura M, Nagamura $Y$, Shin-i T, Abe H, Shimada T, Morishita S, Sasaki T: The genome sequence of silkworm, Bombyx mori. DNA Res 2004, II :27-35.

5. Project HBG: [http://www.hgsc.bcm.tmc.edu/projects/honeybee/].

6. Vaughn JL, Goodwin RH, Tompkins GJ, McCawley P: The establishment of two cell lines from the insect Spodoptera frugiperda (Lepidoptera: Noctuidae). In Vitro 1977, 13:213-2 I7.

7. Huang X, Madan A: CAP3: A DNA Sequence Assembly Program. Genome Research 1999, 9:868-877.

8. Nickerson DA, Tobe VO, Taylor SL: PolyPhred: automating the detection and genotyping of single nucleotide substitutions using fluorescence-based resequencing. Nucleic Acids Res 1997, 25:2745-275।.

9. database ESTMD: [http://www.bioinformatics.ksu.edu:8080/estweb/ index.html].

10. Pashley DP, Ke LD: Sequence evolution in mitochondrial ribosomal and ND-I genes in Lepidoptera: Implications for phylogenetic analyses. Mol Biol Evol 1992, 9: I06 I-I075.

II. Misra S, Crosby MA, Mungall CJ, Matthews BB, Campbell KS, Hradecky P, Huang Y, Kaminker JS, Millburn GH, Prochnik SE, Smith CD, Tupy JL, Whitfied EJ, Bayraktaroglu L, Berman BP, Bettencourt BR, Celniker SE, de Grey AD, Drysdale RA, Harris NL, Richter J, Russo S, Schroeder AJ, Shu SQ, Stapleton M, Yamada C, Ashburner M, Gelbart WM, Rubin GM, Lewis SE: Annotation of the Drosophila melanogaster euchromatic genome: a systematic review. Genome $\mathrm{BiOl}$ 2002, 3:RESEARCH 0083.1-83.22.

12. Landais I, Oligastro M, Mita K, Nohata J, López-Ferber M, DuonorCerutti M, Shimada T, Fournier P, Devauchelle G: Annotation pattern of ESTs from Spodoptera frugiperda Sf9 cells and analysis of the ribosomal protein genes reveal insect-specific features and unexpectedly low codon bias. Bioinformatics 2003 , 19:2343-2350.

13. Altschul SF, Gish W, Miller W, Myers EW, Lipman DJ: Basic local alignment search tool. J Mol Biol 1990, 215:403-410.

14. Genomes BLASTSAS: [http://pistil.ab.a.u-tokyo.ac.jp/kanzen/ blast.html].

15. Ashburner M, Ball CA, Blake JA, Botstein D, Butler H, Cherry JM, Davis AP, Dolinski K, Dwight SS, Eppig JT, Harris MA, Hill DP, IsselTarver L, Kasarskis A, Lewis S, Matese JC, Richardson JE, Ringwald M, Rubin GM, Sherlock G: Gene ontology: tool for the unification of biology. The Gene Ontology Consortium. Nat Genet 2000, 25:25-29.

16. Whitfield CW, Band MR, Bonaldo MF, Kumar CG, Liu L, Pardinas JR, Robertson HM, Soares MB, Robinson GE: Annotated expressed 
sequence tags and cDNA microarrays for studies of brain and behavior in the honey bee. Genome Res 2002, 12:555-566.

17. Ogata H, Goto S, Sato K, Fujibuchi W, Bono H, Kanehisa M: KEGG: Kyoto Encyclopedia of Genes and Genomes. Nucleic Acids Res 1999, 27:29-34.

18. Spodobase: [http://bioweb.ensam.inra.fr/spodobase/].

19. Balu B, Shoue DA, Fraser Jr. MJ, Adams JH: High-efficiency transformation of Plasmodium falciparum by the lepidopteran transposable element piggyBac. Proc Natl Acad Sci USA 2005, 102:1639|-16396.

20. Ding S, Wu X, Li G, Han M, Zhuang Y, Xu T: Efficient transposition of the piggyBac (PB) transposon in mammalian cells and mice. Cell 2005, I 22:473-483.

21. Marcus JM, Ramos DM, Monteiro A: Germline transformation of the butterfly Bicyclus anynana. Proc R Soc Lond B Biol Sci 2004, 27I:S263-S265.

22. Shinmyo Y, Mito T, Matsushita T, Sarashina I, Miyawaki K, Ohuchi H, Noji S: piggyBac-mediated somatic transformation of the two-spotted cricket, Gryllus bimaculatus. Dev Growth Differ 2004, 46:343-349.

23. Bettencourt $R$, Terenius $O$, Faye $I$ : Hemolin gene silencing by dsRNA injected into Cecropia pupae is lethal to next generation embryos. Insect Mol Biol 2002, I I:267-27I.

24. Ewing $B$, Green P: Base-calling of automated sequencer traces using phred. II. Error probabilites. Genome Res 1998, 8: I86-194.

25. Crossmatch: [http://www.sanger.ac.uk/Software/].

26. Chou $\mathrm{HH}$, Holmes $\mathrm{MH}$ : DNA sequence quality trimming and vector removal. Bioinformatics 2001, 17:1093-1 104.

27. RepeatMasker: [http://ftp.genome.washington.edu/].

28. IUBMB: Enzyme nomenclature: Recomendations of the nomenclature committee of the international union of biochemistry and molecular biology. San Diego , Academic Press; 1992.

Publish with Bio Med Central and every scientist can read your work free of charge

"BioMed Central will be the most significant development for disseminating the results of biomedical research in our lifetime. "

Sir Paul Nurse, Cancer Research UK

Your research papers will be:

- available free of charge to the entire biomedical community

- peer reviewed and published immediately upon acceptance

- cited in PubMed and archived on PubMed Central

- yours - you keep the copyright 